\title{
Ultrasensitive Protein Concentration Characterization Based on Weak Measurements
}

\author{
Bowen $\mathrm{SONG}^{1}$, Binglin $\mathrm{CHEN}^{1}$, Chen $\mathrm{YU}^{1}$, and Hua $\mathrm{YANG}^{1,2^{*}}$ \\ ${ }^{1}$ College of Computer Science and Electronic Engineering, Key Laboratory for Micro-/Nano-Optoelectronic Devices of \\ Ministry of Education, Hunan University, Changsha 410082, China \\ ${ }^{2}$ State Key Laboratory of Integrated Optoelectronics, Institute of Semiconductors, Chinese Academy of Sciences, \\ Beijing 100083, China \\ *Corresponding author: Hua YANGＥ-mail: huayang@hnu.edu.cn
}

\begin{abstract}
An optical rotation bio-sensor based on the photonic spin Hall effect was established and applied to detecting the concentration varieties of chiral molecules. The optical rotation, introduced by sample solutions, was exploited to modulate the postselected polarization of a weak measurement system. Much work has been done in the case of glucose and fructose. However, little attention has been paid for biomolecules, such as proteins and amino acids. With this modulation, the optical rotation can be determined through the direction and spin accumulation of light spots, thus mirroring the concentration of solutions. A resolution of $2 \times 10^{-4}$ degree was achieved.
\end{abstract}

Keywords: Weak measurements; the photonic spin Hall effect; optical rotation bio-sensor

Citation: Bowen SONG, Binglin CHEN, Chen YU, and Hua YANG, "Ultrasensitive Protein Concentration Characterization Based on Weak Measurements," Photonic Sensors, 2022, 12(3): 220301.

\section{Introduction}

Chiral molecules, such as protein and amino acid, play such a vital role in body construction [1], immunity [2], maintaining normal system functions [3], etc, so importance has been attached to them. As is known to all, almost every reaction taking place inside the human body is based on or at least associated with chiral molecules. Hence, it is of great significance to precisely determine the concentration of their solutions as well as the countless applications especially in the field of medical biological analysis and drug synthesis [4]. Take proteins as an example, traditional methods of detecting the concentration of protein solutions include Kaiser's nitrogen method [5], Biuret method [6], and Lowry's method [7]. Despite the past success, there still exists a number of problems in use, including questions about the safety as well as time consumption, as these methods are all based on chemical reactions and some of them require high temperature. They are either accurate but less time-consuming or fast but inaccurate. In comparison, this method based on the photonic spin Hall effect (SHE) is of both simplicity and accuracy.

The photonic SHE, which was initially introduced by Onoda et al. [8], is the optical analogy of the electronic spin Hall effect, where the refractive index acts as the electric potential gradient and the spin photons serve as the spin charge. The gradient of refractive index will occur at the interface between two different materials. As the photons pass through the interface, the spin-dependent shift of the light beam centroid will

Received: 3 January 2021 / Revised: 18 August 2021

(C) The Author(s) 2022. This article is published with open access at Springerlink.com

DOI: $10.1007 / \mathrm{s} 13320-022-0644-\mathrm{y}$

Article type: Regular 
appear, which is orthogonal to the refractive index gradient. There has been growing interests in phtonic SHE in experiments $[9,10]$.Over the years, the photonic SHE is greatly lucubrated in diversified physics systems, such as optical physics, high-energy physics [11], semiconductor [12, 13], image processing [14, 15], and system parameter measurement [16-18]. Even as small as the photonic SHE, it is still detectable. In order to detect this subtle shift precisely, the quantum weak measurement was used.

The weak measurement, which was first proposed by Aharonov et al. [19], has been gaining increasing attention as a signal amplification technique recently because of not only observing some subtle physical phenomena but also detecting some physical parameters. Generally, the conventional quantum measurements include a process of strong coupling where the wave function is collapsed. Conventional weak measurement suggests that the coupling between the probe and the observable should be weak and the wave function should stay almost undisturbed. The weak value can be obtained as a simple expression:

$$
\left|A_{w}\right\rangle=\frac{\left\langle\psi_{f}\left|\widehat{\sigma}_{3}\right| \psi_{i}\right\rangle}{\left\langle\psi_{f} \mid \psi_{i}\right\rangle}
$$

where $\left|\psi_{i}\right\rangle$ and $\left|\psi_{f}\right\rangle$ represent the preselected and postselected states, respectively, and $\widehat{\sigma_{3}}$ is an observable value [20, 21].

In the procedure of the weak measurement, the system is preselected as an initial state firstly. Then, the observable is extremely weakly coupled with the pointer state [22]. Finally, the pointer position is recorded when the quantum system is postselected as the final state. However, the weak measurement is valid only under the circumstance that the probe state and the observable are weakly coupled. In some practical situations, it does not always satisfy this condition [23-25]. Hence, the conventional weak measurement needs modification if the coupling is not weak enough. Additionally, when the preselected and postselected states are nearly perpendicular, i.e., $\left\langle\psi_{i} \mid \psi_{f}\right\rangle \rightarrow 0$, the weak value $A_{w}$ would be arbitrarily large. The weak value also needs modification in this situation actually. It is notable that neither strong nor conventional weak measurement is capable of detecting the spin-dependent splitting in the photonic SHE when the wave function is collapsed. Modified weak measurement was used to detect the concentration of protein and amino acid solutions in our work.

The paper is organized as follows. To begin with, the principle of using weak measurement to detect the concentration is discussed. Then, a modified weak measurement is developed, which is for detecting the photonic SHE when the probe wave function is collapsed. Next, the experimental and theoretical results are compared. The results for detecting concentration of different solutions are later shown and analyzed. Finally, a summary is given.

\section{Principle}

The principle of the experiment can be simply illustrated in Fig. 1. The incident light can be divided into two equal-amplitude components that share different directions of polarization, i.e., right and left. Normally, they are centered on the vertical polarization plane and the output beam is still vertically polarized. However, when traveling through optical-active materials, they own unequal speeds due to the structure of the material. This leads to two components owning different reflective indexes which can be defined as $n_{L}$ and $n_{R}$, respectively. As a result, two components are no longer symmetric and the output is no more vertically polarized with the rotation angle $\theta$ in the former polarization plane, when the optical rotation is introduced. $\theta$ can be defined as

$$
\theta=\frac{\pi}{\lambda}\left(n_{L}-n_{R}\right) L
$$

where $\lambda$ is the wavelength, and $L$ represents the distance it goes through. As concentration grows, the 
influence of optical rotation becomes more clear, that is, the displacement of beam centroid is bigger, which can be read out on the charge coupled device (CCD).
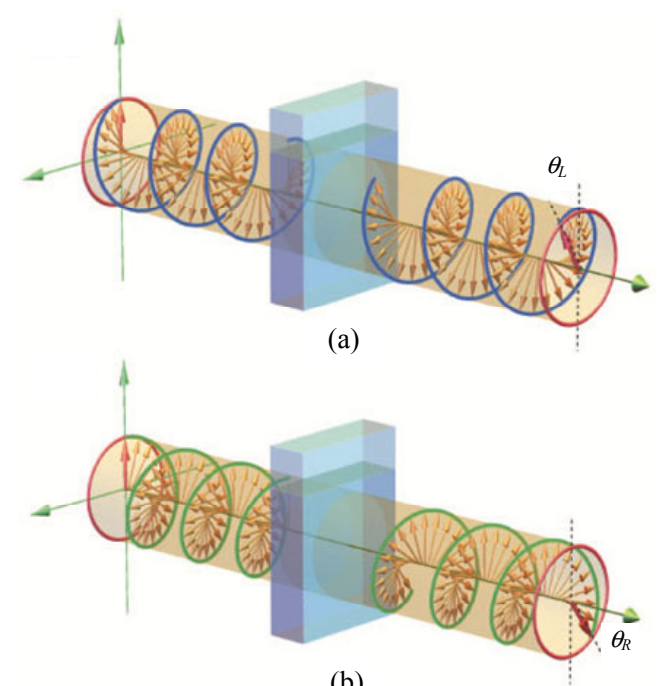

(b)

Fig. 1 Schematic illustrating the change of beam polarization: (a) when the light goes through the right-handed solution, its left-handed polarization changes a comparatively small angle and (b) when the light goes through the right-handed solution, its right-handed polarization changes a bigger angle.

In this section, a modified model of the weak measurement to detect the photonic SHE is developed but firstly, the conventional weak measurement is reviewed. In the process of the weak measurement, an initial state is prepared firstly. After the measuring device is weakly coupled with the system, the observable $\widehat{A_{3}}$ goes through a separate degree interpreted as a meter. After that, the information could be read out from it when postselected section is placed. In this paper, the traverse spatial distribution is used as a meter and the observable is $\widehat{\sigma_{3}}$ and the two eigenstates of the system can be fulfilled with two orthogonal polarizations, namely $|H\rangle$ and $|V\rangle . H$ and $V$ respectively represent horizontal and vertical, $|H\rangle=(|+\rangle+|-\rangle) / \sqrt{2}$ and $|V\rangle=\mathrm{i}(|-\rangle-|+\rangle) / \sqrt{2}$. In this work, the photonic SHE takes place at the air-glass interface. At the very beginning, the $|H\rangle$ is preselected. As only the spatial extent in the $y$ direction is considered, the wave function can be obtained as

$$
\left|\Psi_{\text {initial }}\right\rangle=\int \phi\left(k_{y}\right)\left|k_{y}\right\rangle|H\rangle \mathrm{d} k_{y}
$$

where $k_{y}$ is the wavenumber in the $y$ direction, and $\phi\left(k_{y}\right)$ is the Fourier transform of $\psi(y)$. It is assumed that $\phi\left(k_{y}\right)$ is Gaussian spatial distribution here. The light beam separates into two wave packets of perpendicular states at the interface [26].

$$
\begin{aligned}
& \left|k_{y}\right\rangle|H\rangle \rightarrow \\
& \frac{\left|k_{y}\right\rangle\left[\exp \left(\mathrm{i} k_{y} \delta^{H}\right)|+\rangle+\exp \left(-\mathrm{i} k_{y} \delta^{H}\right)|-\rangle\right]}{\sqrt{2}}
\end{aligned}
$$

where $\delta^{H}$ is defined as $\left(r_{p}+r_{s}\right) \cot \theta_{i} /\left(k_{0} r_{p}\right)$, and $r_{p}$ and $r_{s}$ stand for the Fresnel reflection coefficients for parallel and orthogonal polarizations, respectively. $\theta_{i}$ and $k_{0}$ represent the incident angle and the wavenumber of light in free space, respectively.

Here, the preselected state $\left|\psi_{i}\right\rangle$ is purely the polarization state $|H\rangle$ and the postselected state is $\left|\psi_{f}\right\rangle=|V+\Delta\rangle$, where $\Delta$ represents the postselected angle, namely.

$$
\begin{gathered}
\left|\psi_{i}\right\rangle=|H\rangle \\
\left|\psi_{f}\right\rangle=\sin (-\Delta)|H\rangle+\cos (\Delta)|\psi\rangle .
\end{gathered}
$$

In the spin Hall effect, they become

$$
\begin{gathered}
\left|\psi_{i}\right\rangle=\frac{(|+\rangle+|-\rangle)}{\sqrt{2}} \\
\left|\psi_{f}\right\rangle=-\frac{\mathrm{i}\left(\mathrm{e}^{-\mathrm{i} \Delta}|+\rangle-\mathrm{e}^{\mathrm{i} \Delta}|-\rangle\right)}{\sqrt{2}} .
\end{gathered}
$$

The operator $\hat{A}$ can be written as $\widehat{\sigma_{3}}$ with the right- and left-handed circularly polarization bases.

By calculating the matrix elements, the weak value can be written as $[27,28]$

$$
A_{w}=\frac{\left\langle\psi_{f}\left|\widehat{\sigma_{3}}\right| \psi_{i}\right\rangle}{\left\langle\psi_{f} \mid \psi_{i}\right\rangle}=-\mathrm{i} \cot \Delta .
$$

The result is valid when [27]

$$
\frac{\left|\Delta^{H}\right|}{w} \ll \min [\tan \Delta, \cot \Delta]
$$

where $w$ is the width of the wavefunction.

Generally, the weak value is such a number whose imaginary and real parts respectively correspond to the shifts of momentum and position in wavefunction. It has been experimentally 
manifested in the plasmonic SHE. The pure imaginary part of $A_{w}$ changes the shift of the position displacement $\delta_{H}$ into momentum one.

Next, the free evolution of the wave function before detection is considered. $A_{w}^{H_{\text {con }}}$ is defined as the conventional amplified factor. The displacement can be written as $A_{w}^{H_{\text {con }}}=F\left|A_{w}\right|$ where $F$ is a factor depending on the meter state and free evolution. At a given plane $z$, the free evolution is given by $F=z / z_{r}$, where $z_{r}$ is the Rayleigh length, and eventually, the amplified shift of the conventional weak measurement theory can be obtained as [27]

$$
A_{w}^{H_{\mathrm{con}}} \delta^{H}=\frac{z\left(r_{s}+r_{p}\right) \cot \Delta \cot \theta_{i}}{z_{r} r_{p} k_{0}} .
$$

As the equal above suggests, in the conventional weak measurement, the amplified factor is proportional to the absolute value of weak value.

Note that the analysis discussed above requires very strict conditions to acquire the weak value in the conventional weak measurement theory. In practical experiments of the photonic SHE, $A_{w}$ is extravagantly large when the preselected and postselected states are nearly orthogonal. Hence, the approximation in the conventional theory is not valid under this circumstance [27]: $1-\mathrm{i} k_{y} \delta^{H} A_{w} \approx \mathrm{e}^{-\mathrm{i} k_{y} \delta^{H} A_{w}}$ does not make sense, namely when $\left\langle\psi_{f} \mid \psi_{i}\right\rangle \rightarrow 0$.

In the following section, the modified theory without approximation is developed. In this theory, the measuring procedure of the preselected and postselected ensembles is still effective.

The expected value of the pointer observable $y$, also referred to as the modified amplified shift, can be written as $[18,27]$

$$
\begin{aligned}
& \left\langle y^{H}\right\rangle= \\
& \frac{z\left[2 z_{r} r_{p} k_{0}\left(r_{p}+r_{s}\right)+\chi^{2}\right] \sin (2 \delta) \cot \theta_{i}}{2 z_{r} k_{0}\left(r_{p}+r_{s}\right)^{2} \cos ^{2} \delta \cot ^{2} \theta_{i}+4 z_{r}^{2} r_{p}^{2} k_{0}^{2} \sin ^{2} \Delta}
\end{aligned}
$$

where $\chi=\partial r_{p} / \partial \theta_{i}$. Consequently, the conclusion can be reached that the modified weak measurement theory is needed when detecting the photonic spin
Hall effect with an almost linearly polarized state.

Under the condition of weak coupling, the distribution of the wave function is still Gaussian shape. On the contrary, when preselected and postselected states are exactly or nearly orthogonal, the distortion between left- and right-handed components becomes prominent, thus causing the collapse of the wave function. It leads to the violation of limited conditions when the weak value in the conventional weak measurement is calculated on the assumption that the wavefunction is Gaussian. Therefore, the conventional weak measurement theory is invalid when the quantum system is outside the regime where it is weakly coupled.

In order to verify the correctness of the modified model, experiments were performed. The experimental setup can be shown in Fig. 2. The He-Ne Laser generated a Gaussian beam which went through a short focal length lens (Lens1) and then a polarizer (GLP1), producing a polarized focused beam. When impinging on the prism, the electric fields of two components experienced opposite rotations so as to meet boundary conditions. The incident light was preselected in the $\mathrm{H}$ polarization by $\operatorname{GLP} 1\left(\alpha=0^{\circ}\right)$ and later postselected by GLP2. The prism was placed on a rotation stage in order to control the incident angle $\theta_{i}$.

The experimental and theoretical results are compared in Fig. 3.

The splitting displacement produced by the photonic SHE was extremely small and could not be measured by a macro way. The larger the multiple of the weak measurement method was, the smaller the minimum spot displacement could be observed. That is, the more accurate the measurement would be. As is vividly depicted in the diagram, the amplified displacement was extremely sensitive to the postselected angle $\beta$ when preselected and postselected states were nearly perpendicular to each other. Meanwhile, there was a region that could be defined as the working area, within which the displacement increased in a linear fashion. That was 
why we could detect concentration and varieties of different solutions by recording the beam displacement. With incident angle decreasing, the displacement changed more dramatically. However, the working area kept shrinking as well. In order to make the sensor more sensitive and hold a comparatively large working area, the incident angle of $30^{\circ}$ was chosen. In the experiment, the resolution of the beam displacement observed by the detector CCD was always less than $1 \mu \mathrm{m}$. Therefore, the measurement resolution of optical rotation detection could achieve $2 \times 10^{-4}$ degree in our weak measurement system.

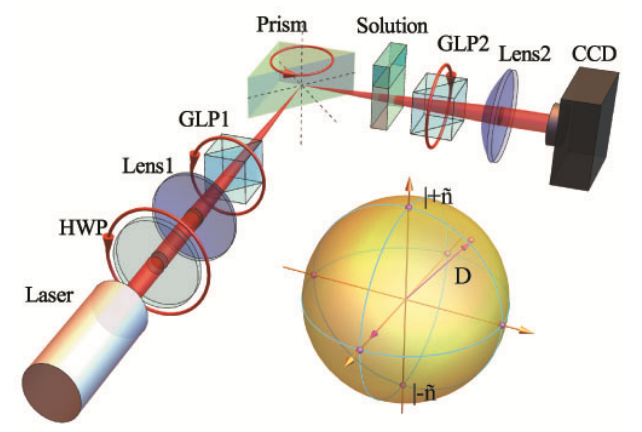

Fig. 2 Experimental setup for characterizing the spin Hall effect of light. He-Ne laser at $632.8 \mathrm{~nm}$; prism with refractive index $n=1.515$; Lens 1 and Lens 2 with effective focal lengths of $50 \mathrm{~mm}$ and $250 \mathrm{~mm}$, respectively; Glan laser polarizers (GLP), GLP1 and GLP2 as well as CCD (Coherent Laser Cam HR). The source of light is at $623.8 \mathrm{~nm}$ (Thorlabs HRP170).

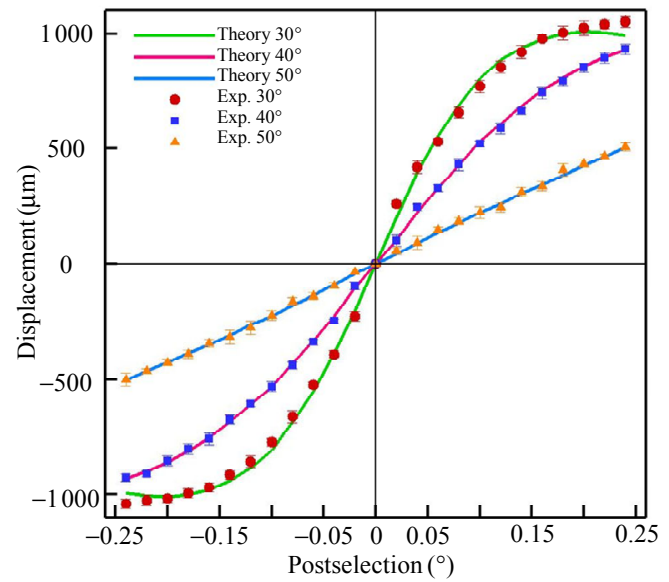

Fig. 3 Experimental and theoretical data at different incident angles of $30^{\circ}, 40^{\circ}$, and $50^{\circ}$. The solid lines and dots represent the theoretical results and experimental data, respectively. We measured the beam displacement every $0.02^{\circ}$ from $-0.24^{\circ}$ to $0.24^{\circ}$.

Proteins and amino acid are essential in organism. Bovine albumin serum (BSA) is widely used in biochemistry, health care product, and buffer. Here, bovine albumin serum fraction V (BSA-V) is taken as the sample. Albumin egg can be used to offer nutrients to embryos and make complete antibodies.

At the beginning of the experiment, the postselected state was adjusted to be orthogonal to the preselected state. In order to demonstrate the capability of concentration detection, cuvettes with different materials and concentrations were introduced into the light path. For BSA-V, the solution at a concentration of $0.5 \mathrm{mg} / \mathrm{mL}$ was made with deionized water. In the same way, BSA-V solutions at different concentrations could be made which was from $0.5 \mathrm{mg} / \mathrm{mL}$ to $6 \mathrm{mg} / \mathrm{mL}$, increasing by $0.5 \mathrm{mg} / \mathrm{mL}$. So were other solutes. After that, the solution was poured into a cuvette which was carefully placed to make it perpendicular to the light. After that, the beam displacement was recorded. Experimental results are shown and compared in Fig. 4.

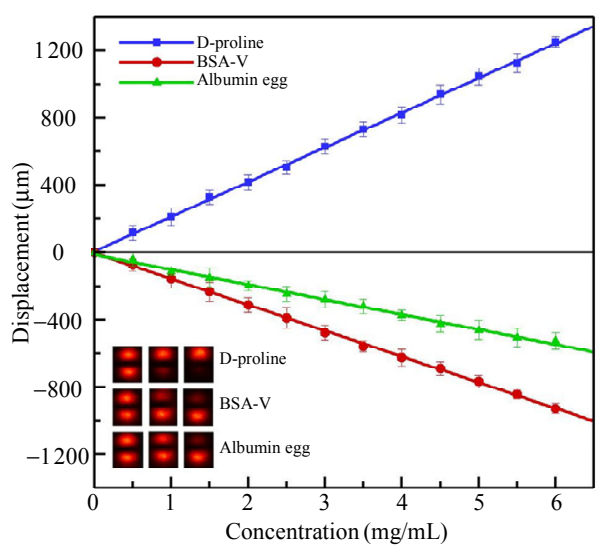

Fig. 4 Results for three samples. The dots are the experimental data. The lines are the fitted curves. Insets exhibit the light spots shown on the CCD of these three samples at the concentration of $0 \mathrm{mg} / \mathrm{mL}, 0.3 \mathrm{mg} / \mathrm{mL}$, and $0.6 \mathrm{mg} / \mathrm{mL}$ from left to right, respectively.

From the diagram, the magnitude of displacements was proportional to the concentration of solutions. A conclusion could be reached that as the concentration increased, the beam displacement under the circumstances of different solutions rose in a linear fashion. It can be seen that the displacement was positive to D-Proline but negative to BSA-V and albumin egg, meaning the chirality of D-Proline was opposite to that of the other two materials. On the other hand, the slopes of different 
curves were disparate. With this calibration curve, the concentration could be obtained easily and fast compared with the traditional chemical methods. Besides, the biosensor was capable of reacting to both left-handed and right-handed materials.

Chirality means that an object cannot coincide with its mirror image. Most proteins in nature are chiral [29]. As a substance that the body produces in the blood to fight disease, antibody is important to the human being, which is a kind of immunoglobulin with chirality [30]. Experiments have proved that the concentration of chiral molecules, whether left-handed or right-handed, could be measured by the weak measurement, so our weak measurement device could also be used to detect the concentration of antibody protein.

\section{Conclusions}

In conclusion, distinguished from the previous work done about glucose and fructose [31-33], an ultrasensitive biosensor based on the photonic spin Hall effect was presented to detect the biomolecule concentration. Different kinds of solutes would cause different directions of polarization and rates of beam displacement's rising. By introducing the quantum weak measurement, the relation between the concentration and spin-Hall shift was established. Firstly, a modified weak measurement theory, compared with the conventional weak measurement theory, was raised and verified by the experiment. Then, the effects of different incident angles were compared and the final incident angle was chosen. Finally, the biosensor was realized and applied to detecting the concentration and kinds of proteins and amino acid. A resolution of $2 \times 10^{-4}$ degree was achieved. In summary, this photonic SHE based on biosensor exhibited great advantages compared with the traditional chemical methods, such as simple operation, high sensitivity, safety, environmental amity as well as low cost. This ultrasensitive biosensor offered a method to fast and precisely detect the concentration and the variety of solutions.
The proposed scheme could be combined with machine learning [34] and extended to frequency domain [35] as well.

\section{Acknowledgment}

The authors gratefully acknowledge the financial support from the National Natural Science Foundation of China (NSFC) (Grant No. 11474089) and the Opened Fund of the State Key Laboratory of Integrated Optoelectronics (Grant No. IOSKL2020KF20).

Open Access This article is distributed under the terms of the Creative Commons Attribution 4.0 International License (http://creativecommons.org/licenses/by/4.0/), which permits unrestricted use, distribution, and reproduction in any medium, provided you give appropriate credit to the original author(s) and the source, provide a link to the Creative Commons license, and indicate if changes were made.

\section{References}

[1] Y. Kim, D. S. Yang, P. Katti, and B. Glancy, "Protein composition of the muscle mitochondrial reticulum during postnatal development," The Journal of Physiology, 2019, 597(10): 2707-2727.

[2] R. Geesala, P. D. Issure, T. Maretzky, and B. Glancy, "Novel functions of inactive rhomboid proteins in immunity and disease," Journal of Leukocyte Biology, 2019, 106(4): 823-835.

[3] J. W. Carbone and S. M. Pasiako, "Dietary protein and muscle mass: translating science to application and health benefit," Nutrients, 2019, 11(5): 1136.

[4] D. Li, T Guan, Y. He, F. Liu, A. Yang, Q. He, et al., "A chiral sensor based on weak measurement for the determination of proline enantiomers in diverse measuring circumstances," Biosensors and Bioelectronics, 2018, 110: 103-109.

[5] H. Wang, N. Pampati, W. M. McCormick, and L. Bhattacharyya, "Protein nitrogen determination by kjeldahl digestion and ion chromatography," Journal of Pharmaceutical Sciences, 2016, 105(6): 1851-1857.

[6] T. E. Dorsey, P. W. McDonald, and O. A. Roels, "A heated biuret-Folin protein assay which gives equal absorbance with different proteins," Analytical Biochemistry, 1977, 78(1): 156-164.

[7] P. J. Geiger and S. P. Bessman, "Protein determination by Lowry's method in the presence of sulfhydryl reagents," Analytical Biochemistry, 1972, 49(2): 467-473. 
[8] M. Onoda, S. Murakami, and N. Nagaosa, "Hall effect of light," Physical Review Letters, 2004, 93(8): 083901.

[9] K. Y. Bliokh, A. Niv, V. Kleiner, and E. Hasman, "Geometrodynamics of spinning light," Nature Photonics, 2008, 2(12): 748-753.

[10] Y. Qin, Y. Li, X. Feng, Z. Liu, H. He, Y. Xiao, et al., "Spin hall effect of reflected light at the air-uniaxial crystal interface," Optics Express, 2010, 18(16): $16832-16839$.

[11] C. A. Dartora, G. G. Cabrera, K. Z. Nobrega, V. F. Montagner, M. H. K. Matielli, F. K. R. De Campos, et al., "Lagrangian Hamiltonian formulation of paraxial optics and applications: study of gauge symmetries and the optical spin Hall effect," Physical Review A, 2011, 83(1): 012110.

[12] S. Murakami, "Dissipationless quantum spin current at room temperature," Science, 2003, 301(5638): $1348-1351$.

[13] M. J. Mattacchione, E. Adam, V. Driel, M. Henry, C. Hautmann, and M. Betz, "Ultrafast optical imaging of the spin Hall effect of light in semiconductors," Physical Review B, 2010, 82(4): 045303.

[14] J. Zhou, H. Qian, C. Chen, J. Zhao, G. Li, Q. Wu, et al., "Optical edge detection based on high-efficiency dielectric metasurface," Proceedings of the National Academy of Sciences, 2019, 116(23): 11137-11140.

[15] S. He, J. Zhou, S. Chen, W. Shu, H. Luo, and S. Wen, "Wavelength-independent optical fully differential operation based on the spin-orbit interaction of light," APL Photonics, 2020, 5(3): 036105.

[16] S. Chen, X. Ling, W. Shu, H. Luo, and S. Wen, "Precision measurement of the optical conductivity of atomically thin crystals via the photonic spin hall effect," Physical Review Applied, 2020, 13(1): 014057.

[17] J. Liu, K. Zeng, W. Xu, S. Chen, H. Luo, and S. Wen, "Ultrasensitive detection of ion concentration based on photonic spin Hall effect," Applied Physics Letters, 2019, 115(25): 251102.

[18] R. Wang, J. Zhou, K. Zeng, S. Chen, X. Ling, W. Shu, et al., "Ultrasensitive and real-time detection of chemical reaction rate based on the photonic spin Hall effect," APL Photonics, 2020, 5(1): 016105 .

[19] Y. Aharonov, D. Z. Albert, and L. Vaidman, "Measurement process in relativistic quantum theory," Physical Review D, 1986, 34(6): 1805.

[20] I. M. Duck, P. M. Stevenson, and E. C. G. Sudarshan, "The sense in which a 'weak measurement' of a spin- $1 / 2$ particle's spin component yields a value 100," Physical Review D, 1989, 40(6): 2112.

[21] N. W. M. Ritchie, J. G. Story, and R. G. Hulet, "Realization of a measurement of a weak value,"
Physical Review Letters, 1991, 66(9): 1107.

[22] C. M. Krowne and J. Q. Shen, "Dressed-state mixed-parity transitions for realizing negative refractive index," Physical Review A, 2009, 79(2): 023818 .

[23] J. Dressel and A. N. Jordan, "Significance of the imaginary part of the weak value," Physical Review A, 2012, 85(1): 012107.

[24] A. Di Lorenzo, "Full counting statistics of weak-value measurement," Physical Review A, 2012, 85(3): 032106.

[25] S. Pang, S. Wu, and Z. B. Chen, "Weak measurement with orthogonal preselection and postselection," Physical Review A, 2012, 86(2): 022112.

[26] H. Luo, X. Zhou, W. Shu, S. Wen, and D. Fan, "Enhanced and switchable spin Hall effect of light near the Brewster angle on reflection," Physical Review A, 2011, 84(4): 043806.

[27] S. Chen, X. Zhou, C. Mi, H. Luo, and S. Wen, "Modified weak measurements for the detection of the photonic spin Hall effect," Physical Review A, 2015, 91(6): 062105.

[28] Y. Wang, S. Chen, S. Wen, and H. Luo, "Realization of ultra-small stress birefringence detection with weak-value amplification technique," Applied Physics Letters, 2021, 118(16): 161104.

[29] J. Maynard and G. Georgiou, "Antibody engineering," Annual Review of Biomedical Engineering, 2000, 2(1): 339-376.

[30] R. S. Cahn, C. Ingold, and V. Prelog, "Specification of molecular chirality," Angewandte Chemie International Edition in English, 1966, 5(4): 385-415.

[31] D. Li, Z. Shen, Y. He, Y. Zhang, Z. Chen, and H. Ma, "Application of quantum weak measurement for glucose concentration detection," Applied Optics, 2016, 55(7): 1697-1702.

[32] X. Qiu, L. Xie, X. Liu, L. Luo, Z. Zhang, and J. Du, "Estimation of optical rotation of chiral molecules with weak measurements," Optics Letters, 2016, 41(17): 4032-4035.

[33] L. Xie, X. Qiu, L. Luo, X. Liu, Z. Li, Z. Zhang, et al., "Quantitative detection of the respective concentrations of chiral compounds with weak measurements," Applied Physics Letters, 2017, 111(19): 191106.

[34] Y. Xu, L. Shi, T. Guan, D. Li, Y. Yang, X. Wang, et al., "Optimization of a quantum weak measurement system with digital filtering technology," Applied Optics, 2018, 57(27): 7956-7966.

[35] D. Li, T. Guan, F. Liu, A. Yang, Y. He, Q. He, et al., "Optical rotation based chirality detection of enantiomers via weak measurement in frequency domain," Applied Physics Letters, 2018, 112(21): 213701. 